
\title{
28 Research Soure \\ Understanding pain neural circuits in a mouse model of depression
}

\author{
Weiwei Yin \\ Lisheng Mei \\ Tingting Sun \\ Yuping Wang \\ Jie Li \\ Changmao Chen \\ Zahra Farzinpour \\ Yu Mao \\ Wenjuan Tao \\ Juan Li \\ Wen Xie \\ Zhi Zhang
}

\section{Video Abstract}

Keywords: Anesthesiology, American Society of Anesthesiologists, University of Science and Technology of China, Anhui Mental Health Center, Anhui Medical University, depression, chronic pain, neuroimaging, central pain processing, chemogenetics, central amygdala, periaqueductal gray, nociception, glutamatergic cells, brain network, neurotransmitter, brain slice, electrophysiology, immunohistochemistry, Hargreaves test

Posted Date: October 23rd, 2020

DOI: https://doi.org/10.21203/rs.3.rs-97501/v1

License: (c) (i) This work is licensed under a Creative Commons Attribution 4.0 International License. Read Full License 


\section{Abstract}

People with depression are more likely to have chronic pain, and people with chronic pain are more likely to be depressed. Those with depression also often report feeling more intense pain, which can lead to a vicious cycle that's hard to break. Scientists, unfortunately, don't have a good understanding of why this happens. But in a new Anesthesiology paper, researchers from China detail a brain circuit in mice that begins to explain this phenomenon - and suggests potential treatments. The team used optogenetics and chemogenetics along with tracing methods to map out neuronal circuits when mice became depressed. In one set of key experiments, mice were restrained in a small tube for 6 hours a day. After 3 weeks of chronic restraint stress, mice began to look depressed. They ate less, showed decreased mobility during a swimming test, and displayed greater sensitivity to noxious stimuli. Compared to controls, the brains of depressed mice showed increased activity in specialized neurons that modulate the inhibitory neurotransmitter GABA. These "GABAergic neurons" occurred in the central amygdala, a major pain processing unit, and projected onto other neurons in the ventrolateral periaqueductal gray, a checkpoint on the pain signaling pathway from the brain to the site of injury. Chronic restraint stress also increased the firing rate of these central amygdala neurons. But inhibiting those neurons didn't lead to reduced responses to noxious experimental stimuli. Unexpectedly, selective inhibition increased sensitivity to noxious stimuli, while selective activation reduced sensitivity. And neither changed the depressive behaviors of the mice. Additional experiments identified a decrease in the firing activity in ventrolateral periaqueductal gray GABAergic neurons in the chronic restraint stress mice compared to controls. Activation of these neurons decreased the animals' tolerance to noxious stimulation, while inhibition increased their tolerance. In contrast, glutamate-modulating neurons in the same area had the opposite response, increasing in activity in the depressed mice. Inhibition of these neurons made the mice more sensitive to noxious stimulation, while activation reduced this sensitivity. Thus, the authors define a brain circuit that operates in depression in which increased inputs from GABAergic neurons from the amygdala disinhibit the periaqueductal gray glutamatergic neurons. With lower excitation of the GABAergic neurons in the central gray, there is less inhibition of the pain-promoting glutamatergic neurons. The findings suggest manipulations targeting this circuit could affect painful symptoms in humans. In particular, it's possible that non-drug approaches, such as deep brain stimulation or transcranial magnetic stimulation, could be effective tools in helping people with depression and chronic pain. 